\title{
Evaluation of two commercial lateral flow devices (LFDs) used for flockside testing of H5N1 highly-pathogenic avian influenza infections in backyard gallinaceous poultry in Egypt
}

\author{
Mohammed Soliman ${ }^{1}$, Abdullah Selim ${ }^{1}$, Vivien J Coward ${ }^{2}$, Mohammed K Hassan ${ }^{1}$, Mona M Aly ${ }^{1}$, \\ Jill Banks² and Marek J Slomka ${ }^{2} *$ \\ ${ }^{1}$ National Laboratory for Veterinary Quality Control on Poultry Production, Animal Health Research Institute, Ministry \\ of Agriculture, PO Box 264, Nadi Elseid Street, Dokki, Giza, Egypt, ${ }^{2}$ Avian Virology, Building 64, Veterinary \\ Laboratories Agency (VLA-Weybridge), Woodham Lane, Addlestone, Surrey, KT15 3NB, United Kingdom \\ *Correspondence to: Marek Slomka, Email: m.slomka@vla.defra.gsi.gov.uk, Tel: +44 1932 341111, Fax: +44 1932 \\ 357239
}

Received 30 June 2010, Revised 29 September 2010, Accepted 30 September 2010, Published online 13 October 2010

J Mol Genet Med (2010), 4, 247-251

(C) Crown copyright: This article is being published and displayed under the terms and conditions of the Click-Use Licence. The article or its contents may be reproduced by obtaining a PSI Click-Use Licence at http://www.opsi.gov.uk/click-use/system/online/pLogin.asp.

\begin{abstract}
Quickvue and Anigen lateral flow devices (LFDs) were evaluated for detection of H5N1 highly pathogenic avian influenza (HPAI) infections in Egyptian poultry. Sixty five chickens and two turkeys were sampled in eight flocks where H5N1 HPAI infection was suspected. Swabs (tracheal and cloacal) and feathers were collected from each bird for flockside testing by the two LFDs. The same clinical specimens were transported for laboratory testing by M gene RRT PCR where a positive result by this "gold standard" test for one or both swabs from a given bird indicated infection at the bird level, showing 57 birds (including 15 carcassess) to be truly AI infected. Among these 57, similar bird-level LFD testing of swabs showed 43 and 44 to be AI infected by Quickvue and Anigen LFDs, respectively. Nine birds were AI negative by M gene RRT PCR and both LFDs, and one was M gene RRT PCR negative but positive by both LFDs, suggesting one false positive LFD result. Sensitivities of the LFDs relative to M gene RRT PCR were $77.2 \%$ for Anigen and $75.4 \%$ for Quickvue tests, with $90.0 \%$ specificity for both. By including feathers with swabs for LFD testing, the number of LFD positives among 57 infected birds increased by four to 48 by Anigen and 47 by Quickvue, increasing the sensitivity of the LFDs to $84.2 \%$ and $82.5 \%$ for Anigen and Quickvue, respectively. Although LFD sensitivity cannot compare to the high sensitivity displayed by validated AI RRT PCRs, they may be utilised for flockside testing of birds infected with HPAI at the peak of viral shedding, when birds are displaying advanced clinical signs or sampled as fresh carcasses. Swabs are classic field specimens collected from outbreaks, but inclusion of feathers from birds infected with H5N1 HPAI increased LFD sensitivity. However, the LFD false positive observation emphasises the importance of returning samples for confirmatory laboratory testing.
\end{abstract}

KEYWORDS: Lateral flow device (LFD), H5N1 HPAI, flockside

\section{INTRODUCTION}

Interest in rapid flockside diagnosis of avian influenza (AI) infections in poultry has increased in recent years, particularly following the emergence and extensive spread of H5N1 highly pathogenic (HP) AI (Alexander, 2007;
Brown, 2010). H5N1 HPAI is a continuing epizootic, which has become endemic in some countries while public health remains a concern (Capua and Alexander, 2007). Laboratory methods for AI diagnosis are outlined in the European Union (EU) and the World Organisation for Animal Health (OIE) Diagnostic Manuals, in which virus 
isolation (VI) in embryonated fowl eggs continues to serve as the gold standard (EU, 2006; OIE, 2009). Real-time reverse transcription-PCR (RRT-PCR) has emerged as a rapid, highly-sensitive and specific tool for AI laboratory diagnosis (Hoffmann et al, 2009), and optimised protocols are at least as sensitive as VI (Slomka et al, 2010).

However, the potential rapidity with which HPAI outbreaks may spread remains a concern. Several commercial lateral flow device (LFD) tests are available for rapid flockside testing of AI. LFD evaluations have been described using field specimens collected during H5N1 HPAI outbreaks (Chua et al, 2007; Loth et al, 2008) and from experimentally-infected birds (Marché and van den Berg, 2010). In Egypt, H5N1 HPAI first occurred in 2006 (Aly et al, 2008) and is now considered endemic (Cattoli et al, 2009). This study describes the evaluation of two commercial AI LFDs in Egypt during 2009, and focused on suspected H5N1 HPAI outbreaks in backyard poultry, from which tracheal and cloacal swabs were collected and tested by LFDs in the field. Tracheal and cloacal swabs were also collected from the same birds for testing by $M$ gene RRT-PCR in order to provide a highlysensitive and specific determination of whether they were truly AI infected. Feathers collected from all swabbed birds were also tested by LFDs and $M$ gene RRT-PCR.

\section{MATERIALS AND METHODS}

\section{Lateral flow devices (LFDs)}

The Quickvue Influenza Test (Quidel) and the AIV Ag Test kit (Anigen) were used following the manufacturers' protocols. Feather samples were similarly expressed into the kits’ LFD extraction buffers for testing.

\section{Samples from poultry flocks}

Swabs (tracheal and cloacal) and feathers were collected between February and April 2009 in Egypt from 65 chickens and two turkeys from eight backyard poultry flocks in which clinical signs were observed with suspicion of H5N1 HPAI infection. Chickens included 15 carcasses of recently dead birds from four flocks. No apparently healthy flocks were sampled in this study.

\section{Sampling protocol in the field}

Six swabs were taken from each bird, consisting of three from the trachea and three from the cloaca. The order of swabbing was carefully noted. Young feathers (maximum $4-5 \mathrm{~cm}$ long) of immature character, which possessed calami that had not calcified, were also collected from each bird. Swabs were tested by the two AI LFDs. For the first bird the first swabs (tracheal and cloacal) were tested by the Quickvue LFD, while the second swabs (tracheal and cloacal) were tested by the Anigen LFD. For the next bird the order of swab testing was reversed for the two AI LFDs. This alternating approach was continued throughout for all sampled birds. Flockside LFD testing was conducted at the location of the suspected outbreaks. The third swabs (tracheal and cloacal) taken from each bird were transported on ice on the same day to the laboratory at NLQP, Dokki, Egypt, where $1 \mathrm{ml}$ of brain heart infusion broth containing antibiotics (BHIB) was added. Feathers from each bird were divided into three portions; two lots of 2-3 feather calami were tested flockside by each LFD. Each third feather portion was transported to the laboratory where they were expressed in 1ml BHIB. All BHIB fluids were stored frozen at -80C until required for RNA extraction.

RNA extraction, AI RRT-PCRs, virus isolation and typing

RNA was extracted from $140 \mu$ l of BHIB as previously described (Slomka et al, 2007). $M$ gene RRT-PCR (Spackman et al, 2002) was used as recommended in the EU AI Diagnostic Manual (EU, 2006) to provide "gold standard" AI qualitative results in comparison to corresponding LFD results as described by Loth et al (2008). H5N1 infection was confirmed by a combination of VI, classical subtyping and H5 RRT-PCR (EU, 2006; OIE, 2009; Slomka et al, 2007).

\section{RESULTS}

\section{AI infected birds by $M$ gene RRT-PCR}

Sixty-seven birds from eight backyard flocks were assessed as to whether they were positive for AI infection on the basis of testing tracheal and cloacal swabs, this being the normal sampling approach during AI outbreak investigations (OIE, 2009). An individual bird was considered infected if either or both swabs were positive by $M$ gene RRT-PCR. By this test 57 birds (56 chickens and one turkey) were diagnosed to be truly AI infected, including 15 chicken carcasses (Figure 1). Testing of feathers by $M$ gene RRT PCR did not reveal any additional AI-infected birds. Hence we did not find any birds that were $\mathrm{M}$ gene RRT PCR negative on swabs but positive on feathers. H5N1 infection was confirmed in seven flocks, with one being AI negative.

\section{AI LFD comparison to M gene RRT PCR}

This was made between results obtained for individual birds by each of the two LFDs and the $M$ gene RRT-PCR, initially on the basis of testing two swabs per bird. Of the 42 live infected birds, 28 were also positive by the Quickvue LFD. These 28 birds plus one additional chicken were found positive by the Anigen LFD (Figure 1A). The 15 carcasses were AI positive by both LFDs and the $M$ gene RRT-PCR (Figure 1A). Nine birds were negative by LFDs (both tests) and $M$ gene RRT-PCR. One bird was considered to be a false-positive by both AI LFDs as it was $M$ gene RRT-PCR negative (Figure 1A). Comparison of these methods for swabs revealed that for individual birds (live and carcasses) the Anigen and Quickvue LFD sensitivities were $77.2 \%$ and $75.4 \%$, respectively (Table $1 \mathrm{~A}$ ).

When feather samples were included as a third clinical specimen, the numbers of true positive live birds identified by LFDs increased to 33 and 32 for the Anigen and Quickvue tests, respectively (compare Figure 1A with 1B). Therefore, testing of three specimens as opposed to two swabs increased the number of LFD positive birds by four for each LFD. When tested by swabs only, these four chickens were all $M$ gene RRT-PCR positive by one or both swabs, but LFD negative by both swabs (see Figure $1 \mathrm{~A}$ and $1 \mathrm{~B}$ ). Inclusion of feathers with swabs from all birds (live and carcasses) increased LFD sensitivity to 
A. Swabs only

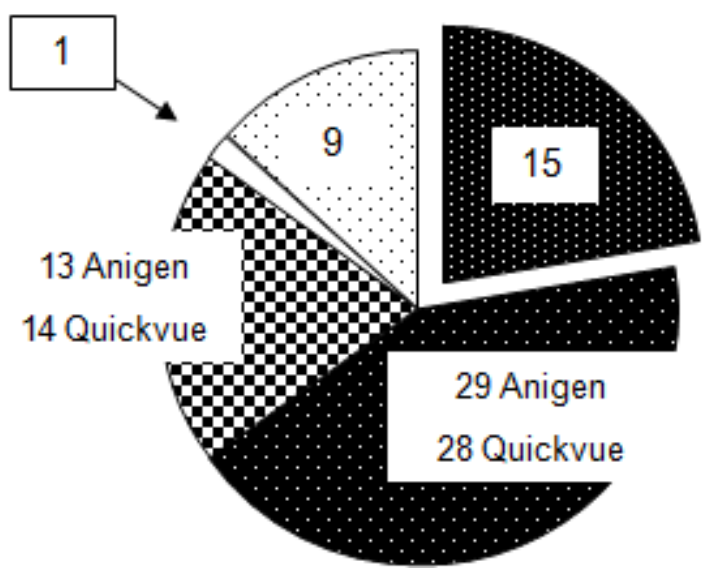

B. Swabs and feathers

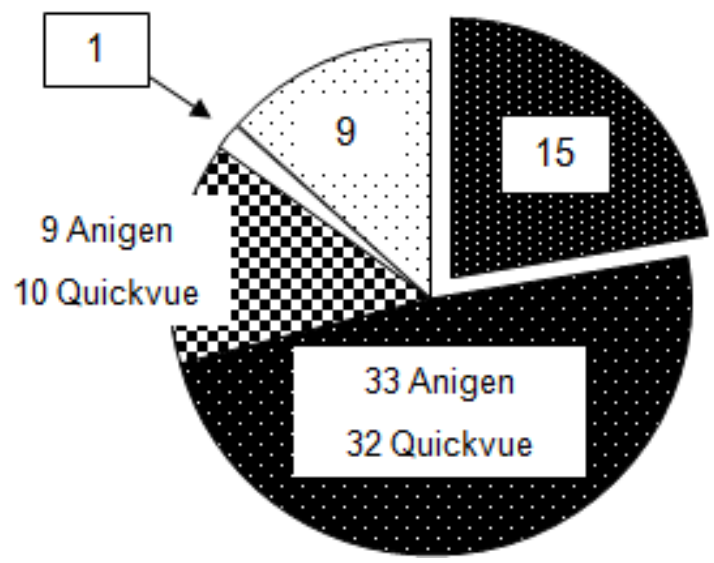

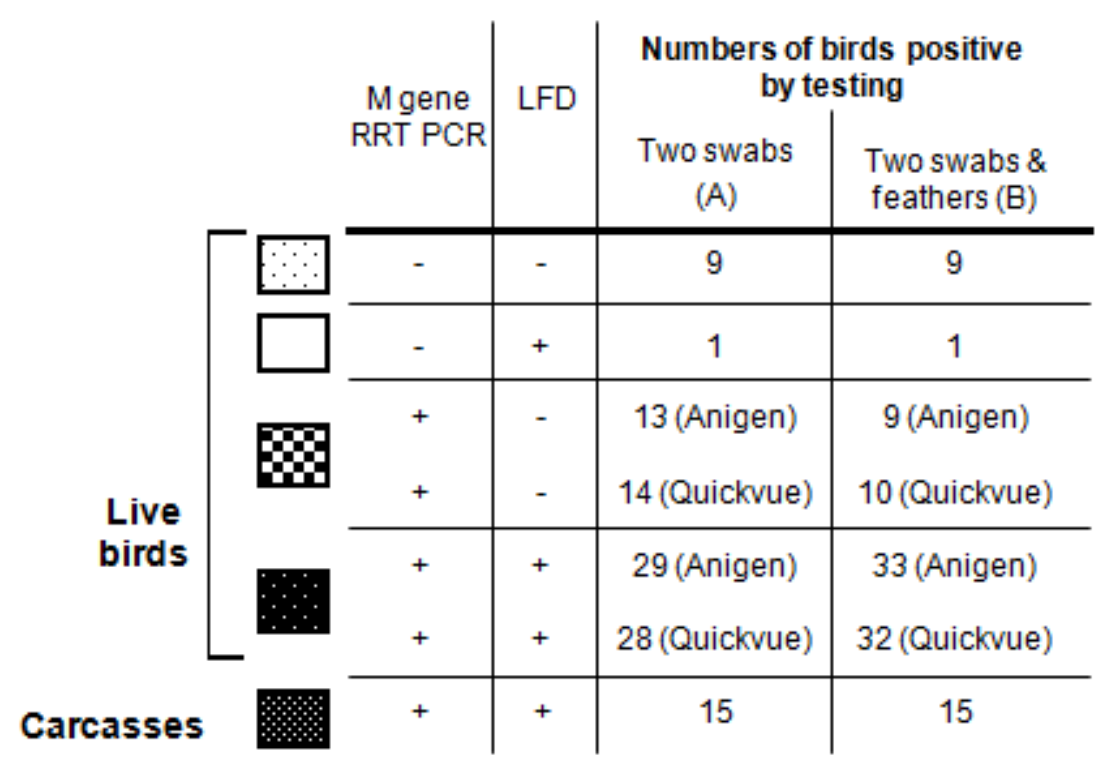

Figure 1. Diagnosis of AI infection in 65 chickens and two turkeys at the individual bird level by M gene RRT PCR and LFDs. Five categories of test result at the bird level are illustrated. For a given bird to have been considered positive by a given test, at least one of the clinical specimens had given a positive result. A. Presents bird-level results on the basis of testing swabs only, while (B) presents bird-level results from the testing of swabs and feathers. The segment indicating 15 carcasses is shown detached from the four other segments that contain 52 live birds from diseased flocks.

84.2\% and 82.5\% for Anigen and Quickvue LFDs, respectively (Table $1 \mathrm{~B})$.

The specificity for each LFD was $90 \%$ for testing two swabs with or without feathers (Table $1 \mathrm{~A}$ and $1 \mathrm{~B}$ ). However, the relatively small number (10) of true AI negative birds in the study population, identified as such by $M$ gene RRT-PCR, was reflected in the broad $95 \%$ confidence intervals (CIs; Table 1A and 1B).

\section{Comparison of the type of clinical specimens}

Diagnostic sensitivity and specificity of the LFDs was determined separately for each type of specimen regardless of the test results for the other two clinical specimens from the same bird (Table 2). For tracheal swabs, both LFDs displayed a sensitivity of $69.8 \%$ and specificity of $92.9 \%$ (Table 2A). For cloacal swabs, the Anigen LFD had a sensitivity of $76.5 \%$ and a specificity of
87.5\%, while the Quickvue LFD had a sensitivity of 75\% and specificity of $100 \%$ (Table $2 \mathrm{~B}$ ). For feather samples, a lower sensitivity of $65.1 \%$ was observed with a specificity of $91.7 \%$ for both LFDs (Table 2C).

\section{DISCUSSION}

Several commercial LFDs for AI detection have emerged in the veterinary diagnostics market in recent years that could be employed for rapid flockside testing of poultry. This methodology has the potential of saving time and accelerates important decision-making processes necessary for effective AI outbreak management. However, there has been a paucity of published validation data concerning LFD test performance. Emergence of the continuing H5N1 HPAI epidemic, initially in Asia followed by spread to Europe and Africa, has coincided with an interest in what role LFDs may play in local outbreak management. It 
Table 1. Diagnostic sensitivity and specificity calculations for LFD tests relative to $M$ gene RRT-PCR determined at the level of individual birds.

A:
\begin{tabular}{|c|c|c|c|c|}
\hline $\begin{array}{c}\text { n=67; on the } \\
\text { basis of testing } \\
\text { two swabs }\end{array}$ & \multicolumn{2}{|c|}{$\begin{array}{c}\text { M gene RRT-PCR } \\
+\end{array}$} & - & Total \\
\hline \multirow{3}{*}{ LFDs } & + & $\begin{array}{c}44 \text { (Anigen) } \\
43 \text { (Quickvue) }\end{array}$ & 1 & $\begin{array}{c}45 \text { (Anigen) } \\
44 \text { (Quickvue) }\end{array}$ \\
\cline { 2 - 5 } & - & $\begin{array}{c}13 \text { (Anigen) } \\
14 \text { (Quickvue) }\end{array}$ & 9 & $\begin{array}{c}22 \text { (Anigen) } \\
23 \text { (Quickvue) }\end{array}$ \\
\hline Total & 57 & 10 & 67 \\
\hline
\end{tabular}

Sensitivity: Anigen: 44/44+13 = 77.2\% (95\% CI: 64.2-87.3\%) Quickvue: 43/43+14= 75.4\% (95\% CI: 62.2-85.9\%)

Specificity (both AI LFDs): 9/1+9 = 90.0\% (95\% CI: 55.5-99.8\%)

B:

\begin{tabular}{|c|c|c|c|c|}
\hline \multicolumn{1}{|c|}{$\begin{array}{c}\text { n= 67, on the } \\
\text { basis of testing } \\
\text { two swabs \& } \\
\text { feathers }\end{array}$} & \multicolumn{2}{|c|}{$\begin{array}{c}\text { M gene RRT-PCR } \\
+\end{array}$} & - & Total \\
\hline \multirow{2}{*}{ LFDs } & + & $\begin{array}{c}48 \text { (Anigen) } \\
47 \text { (Quickvue) }\end{array}$ & 1 & $\begin{array}{c}49 \text { (Anigen) } \\
48 \text { (Quickvue) }\end{array}$ \\
\cline { 2 - 5 } & - & $\begin{array}{c}9 \text { (Anigen) } \\
10 \text { (Quickvue) }\end{array}$ & 9 & $\begin{array}{c}18 \text { (Anigen) } \\
19 \text { (Quickvue) }\end{array}$ \\
\hline Total & 57 & 10 & 67 \\
\hline
\end{tabular}

Sensitivity: Anigen: 48/48+9 = 84.2\% (95\% CI: 72.1-92.5\%)

Quickvue: $47 / 47+10=82.5 \%$ (95\% CI: 70.1-91.2\%)

Specificity (both AI LFDs): 9/1+9 = 90.0\% (95\% CI: 55.5-99.8\%)

Table 2. Diagnostic sensitivity and specificity calculations for LFD tests relative to $M$ gene RRT-PCR, determined separately for each of the three clinical specimens.

A:

\begin{tabular}{|l|c|c|c|c|}
\hline $\begin{array}{c}\text { A: } \\
\text { tracheal swabs } \\
\text { alone }\end{array}$ & \multicolumn{2}{|c|}{$\begin{array}{c}\text { M gene RRT-PCR } \\
\text { Total }\end{array}$} \\
\hline \multirow{2}{*}{ LFDs } & + & 37 & 1 & 38 \\
\cline { 2 - 5 } & - & 16 & 13 & 29 \\
\hline \multicolumn{2}{|c|}{ Total } & 53 & 14 & 67 \\
\hline
\end{tabular}

Sensitivity: $37 / 37+16=69.8 \%$ (95\% CI: $55.7-81.7 \%)$

Specificity: 13/1+13 = 92.9\% (95\% CI: 66.1-99.8\%)

B:

\begin{tabular}{|c|c|c|c|c|}
\hline \multirow{2}{*}{\multicolumn{2}{|c|}{$\begin{array}{l}\mathrm{n}=67, \text { cloacal } \\
\text { swabs alone }\end{array}$}} & \multicolumn{2}{|c|}{$M$ gene RRT-PCR } & \multirow{2}{*}{ Total } \\
\hline & & + & - & \\
\hline \multirow{2}{*}{ LFDs } & + & 39 & $\begin{array}{c}2 \text { (Anigen) } \\
0 \text { (Quickvue) }\end{array}$ & $\begin{array}{l}41 \text { (Anigen) } \\
39 \text { (Quickvue) }\end{array}$ \\
\hline & - & $\begin{array}{c}12 \text { (Anigen) } \\
13 \text { (Quickvue) }\end{array}$ & $\begin{array}{c}14 \text { (Anigen) } \\
15 \text { (Quickvue) }\end{array}$ & $\begin{array}{l}26 \text { (Anigen) } \\
28 \text { (Quickvue) }\end{array}$ \\
\hline \multicolumn{2}{|l|}{ Total } & $\begin{array}{c}51 \text { (Anigen) } \\
52 \text { (Quickvue) }\end{array}$ & $\begin{array}{c}16 \text { (Anigen) } \\
15 \text { (Quickvue) }\end{array}$ & 67 \\
\hline
\end{tabular}

Sensitivity: Anigen: 39/39+12 = 76.5\% (95\% CI: 62.5-87.2\%) Quickvue: 39/39+13 = 75.0\% (95\% CI: 61.1-86.0\%)

Specificity: Anigen: 14/2+14 = 87.5\% (95\% CI: 61.7-98.5\%) Quickvue: 15/0+15 = 100\% (95\% CI: 78.2-100\%)

C:

\begin{tabular}{|c|c|c|c|c|}
\hline \multicolumn{2}{|c|}{$\begin{array}{c}\mathbf{n}=\mathbf{6 7} \\
\text { feathers alone }\end{array}$} & \multicolumn{2}{|c|}{$\begin{array}{c}\text { M gene RRT-PCR } \\
+\end{array}$} & Total \\
\hline \multirow{2}{*}{ LFDs } & + & 28 & 2 & 30 \\
\cline { 2 - 5 } & - & 15 & 22 & 37 \\
\hline \multicolumn{2}{|c|}{ Total } & 43 & 24 & 67 \\
\hline
\end{tabular}

Sensitivity: 28/28+15 = 65.1\% (95\% CI: 49.1-79.0\%)

Specificity: 22/2+22 = 91.7\% (95\% CI: 73.0-99.0\%) must be emphasised that the OIE Diagnostic Manual for AI currently does not recommend LFDs for AI diagnosis and is unlikely to do so until rigorous and robust validation data accumulates, which may indicate in which particular AI infection situations LFDs may have a role (OIE, 2009).

The analytical sensitivity limit of the Anigen and Quickvue LFDs was shown to be approximately 10,000 $50 \%$ egg-infectious doses $\left(\mathrm{EID}_{50}\right) / \mathrm{ml}$ (data not shown), which is at least three orders of magnitude less than the detection limit of optimised highly-sensitive AI RRTPCRs (Spackman et al, 2002; Slomka et al, 2007; Slomka et al, 2009). Despite the sensitivity limitations of LFDs, examination of AI RRT-PCR data obtained during outbreak investigations suggested that LFDs may be sufficiently sensitive to give positive AI results with hightitre swabs obtained from diseased or recently-dead H5N1 HPAI infected galliformes (Slomka et al, 2010). The endemic H5N1 HPAI poultry situation in Egypt provided a setting where LFDs could be evaluated prospectively through flockside testing.

Loth et al (2008) tested only oro-pharyngeal swabs from 155 chickens sampled in diseased back-yard flocks in Indonesia by the Anigen and FluDetect (Synbiotics) LFDs, and reported a sensitivity of $69 \%$ and $71 \%$, respectively, relative to $M$ gene RRT-PCR. This is a very similar finding to the $69.8 \%$ sensitivity for the Anigen and Quickvue LFD testing of tracheal swabs from chickens and turkeys in Egypt (Table 2A) where flocks were similarly sampled on suspicion of HPAI. However, the current study showed that if sampling included more clinical specimens per bird, test sensitivity increased from $69.8 \%$ (both LFDs) for tracheal swabs alone (Table 2A), to $77.2 \%$ (Anigen) and $75.4 \%$ (Quickvue) for tracheal swabs plus cloacal swabs, to $84.2 \%$ (Anigen) and $82.5 \%$ (Quickvue) for swabs and feathers (Tables 1).

Testing of chicken and turkey swabs plus feathers from H5N1 HPAI infected flocks demonstrated that the two LFDs can correctly identify a significant number of true AI infections, provided that swabs are collected from birds that are either recently dead due to HPAI or displaying advanced clinical signs of HPAI disease, when it is known that respiratory and enteric shedding of HPAI is at maximum titre (Slomka et al, 2010). It may be more cost-effective to collect only one type of swab, provided that sufficient numbers of birds are sampled per flock (Loth et al, 2008). It is unlikely that LFDs would detect H5N1 HPAI shedding in infected gallinaceous poultry sampled at the pre-clinical stage, as much lower titres of AI are observed at the early stage of HPAI infection (Slomka et al, 2010). This has been demonstrated in a recent study which included LFD testing of swabs from H5N1 HPAI experimentallyinfected chickens at the early pre-clinical stage (Marché and van den Berg, 2010).

A specificity of $90 \%$ was observed for LFD testing of individual Egyptian birds by testing two swabs with or without feathers (Table 1). The broad CI range reflected the relatively small numbers of true uninfected (i.e., $M$ gene RRT-PCR-negative) chickens in the study population 
(10 birds), which excluded rigorous assessment of LFD specificity. However, the $90 \%$ specificity underlined the importance of confirming that flocks positive by LFD flockside testing are positive by laboratory testing, for which the proven sensitivity and specificity of VI and AI RRT-PCR provides a more accurate result. Some LFD false positives were also observed in the Indonesian LFD evaluation, where the Anigen LFD had 98\% specificity (Loth et al, 2008).

\section{CONCLUSIONS}

- Although not highly sensitive, LFDs can successfully detect HPAI shedding in diseased gallinaceous poultry.

- Advantages of flockside testing

- Testing feathers in addition to swabs from HPAI infected chickens and turkeys increases the sensitivity of the LFDs.

- Clinical specimens still need to be sent to the laboratory for confirmatory testing by more sensitive and specific methods.

\section{ACKNOWLEDGEMENTS}

The authors wish to thank the European Union Framework 6 Programme (EU FP6) for funding the EU FLUAID and FLUTEST projects which supported this collaborative study. Comments on this manuscript from Dennis Alexander are also gratefully acknowledged.

\section{COMPETING INTERESTS}

None declared.

\section{LIST OF ABBREVIATIONS}

AI; avian influenza

CI; confidence intervals

EID $_{50} ; 50 \%$ egg-infectious doses

EU; European Union

HPAI; highly-pathogenic avian influenza

LFD; lateral flow device

OIE; World Organisation for Animal Health

RRT-PCR; real-time reverse transcription polymerase chain

reaction

VI; virus isolation

\section{REFERENCES}

Alexander DJ. 2007. An overview of the epidemiology of avian influenza. Vaccine, 25, 5637-5644.

Aly MM, Arafa A and Hassan MK. 2008. Epidemiological findings of outbreaks of disease caused by highly pathogenic H5N1 avian influenza virus in poultry in Egypt during 2006. Avian Dis, 52, 269-277.

Brown IH. 2010. Summary of avian influenza activity in Europe, Asia, and Africa, 2006-2009. Avian Dis, 54, 187-193.

Capua I and Alexander DJ. 2007. Animal and human health implications of avian influenza infections. Biosci Rep, 27, 359-372.

Cattoli G, Monne I, Fusaro A et al. 2009. Highly pathogenic avian influenza virus subtype $\mathrm{H} 5 \mathrm{~N} 1$ in Africa: A comprehensive phylogenetic analysis and molecular characterization of isolates. PLoS ONE, 4, e4842.

Chua T-H, Ellis TM, Wong CW et al. 2007. Performance evaluation of five detection tests for avian influenza antigen with various avian samples. Avian Dis, 51, 96-105.

EU (European Union). Diagnostic manual for avian influenza [Internet]. 2006. [cited 2009 Nov 23]. Official Journal of European Union L237/1 (31Aug). URL: http://eurlex.europa.eu/LexUriServ/site/en/oj/2006/1_237/l_2372006083 1en00010027.pdf

Hoffmann B, Beer M, Reid SM et al. 2009. A review of RT-PCR technologies used in veterinary virology and disease control: sensitive and specific diagnosis of five livestock diseases notifiable to the World Organisation for Animal Health. Vet Microbiol, 139, 1-23.

Loth L, Prijono WD, Wibawa H and Usman TB. 2008. Evaluation of two avian influenza type A rapid antigen tests under Indonesian field conditions. J Vet Diagn Invest, 20, 642-644.

Marché S and van den Berg T. 2010. Evaluation of rapid antigen detection kits for the diagnosis of highly pathogenic avian influenza H5N1 infection. Avian Dis, 54, 650-654.

OIE (World Organisation for Animal Health). Avian influenza: Manual of Diagnostic Tests and Vaccines for Terrestrial Animals (2009), Chapter 2.3.4. Paris: OIE. URL: http://www.oie.int/eng/ normes/mmanual/2008/pdf/2.03.04_AI.pdf

Slomka M J, Pavlidis T, Banks J et al. 2007. Validated H5 Eurasian real-time PCR and its application in H5N1 outbreaks in 2005-2006. Avian Dis, 51, 373-377.

Slomka MJ, Pavlidis T, Coward V J et al. 2009. Validated RealTime reverse transcriptase PCR methods for the diagnosis and pathotyping of Eurasian $\mathrm{H} 7$ avian influenza viruses. Influenza Other Respir Viruses, 3, 151-164.

Slomka MJ, Irvine RM, Pavlidis T, Banks J and Brown IH. 2010. Role of Real-Time RT-PCR platform technology in the diagnosis and management of notifiable avian influenza outbreaks: Experiences in Great Britain. Avian Dis, 54, 591-596.

Spackman E, Senne DA, Myers TJ et al. 2002. Development of a real-time reverse transcriptase PCR assay for type A influenza virus and the avian $\mathrm{H} 5$ and $\mathrm{H} 7$ hemagglutinin subtypes. J Clin Microbiol, 40, 3256-3260. 